\title{
A Rare Complication of Antibiotic (Piperacillin/Tazobactam) Therapy: Resistant Hypokalemia
}

\author{
Faruk Kutluturk ${ }^{\mathrm{a}, \mathrm{d}}$, Suheyla Uzun ${ }^{\mathrm{b}}$, Turker Tasliyurt ${ }^{\mathrm{b}}$, Safak Sahin ${ }^{\mathrm{b}}$, \\ Sener Barut ${ }^{\mathrm{c}}$, Banu Ozturk ${ }^{\mathrm{b}}$, Abdulkerim Yilmaz ${ }^{\mathrm{b}}$
}

\begin{abstract}
Piperacillin/tazobactam is a commonly used antibiotic with tolerable side effects and broad antimicrobial activity in general practice. Here in we report a patient with resistant and severe hypokalemia induced by piperacillin/tazobactam. There were no other underlying renal or hepatic illness and other causes of hypokalemia. When the treatment was stopped, hypokalemia resolved immediately without potassium replacement. We also evaluated the causality of the case report. We concluded this causality as probable/likely category according to WHO-UMC Causality Categories. We concluded that piperacillin/tazobactam may be cause severe hypokalemia and we must be careful to monitor of serum electrolytes especially potassium during antibiotic treatment.
\end{abstract}

Keywords: Resistant hypokalemia; Piperacillin/tazobactam; Antibiotherapy

\section{Introduction}

Hypokalemia, which is a common electrolyte abnormality, is categorized mild and severe hypokalemia when serum potassium level is $3.0-3.5 \mathrm{mmol} / \mathrm{L}$ and less than $2.5 \mathrm{mmol} / \mathrm{L}$, respectively. Mild hypokalemia is often asymptomatic. Severe hypokalemia is associated with generalized weakness, rhabdomyolysis and paralysis. Both mild and severe hypo-

\footnotetext{
Manuscript accepted for publication July 10, 2012

${ }^{a}$ Department of Endocrinology and Metabolism, Gaziosmanpasa University, Faculty of Medicine, Tokat, Turkey

${ }^{\mathrm{b}}$ Department of Internal Medicine, Gaziosmanpasa University, Faculty of Medicine, Tokat, Turkey

${ }^{\mathrm{c}}$ Department of Infection Disease, Gaziosmanpasa University, Faculty of Medicine, Tokat, Turkey

${ }^{\mathrm{d}}$ Corresponding author: Faruk Kutluturk, Department of Internal Medicine, Division of Endocrinology and Metabolism,

Gaziosmanpasa University, Faculty of Medicine, 60100, Tokat,

Turkey.Email: fkutluturk@yahoo.com
}

doi: http://dx.doi.org/10.4021/jmc799w kalemia can increase the incidence of cardiac arrhythmias. Patients in the intensive care unit are at increased risk for hypokalemia due to renal dysfunction, various medications [1]. Piperacillin/tazobactam, a broad-spectrum antibiotic, is frequently used as an empirical treatment. According to our knowledge, there were only two case reports about piperacillin induced hipokalemia in the literature. We report a case of resistant hypokalemia induced by piperacillin/tazobactam.

\section{Case Report}

An 83-year-old male patient was admitted to hospital with the postoperatively infection of incision area after left hipbone revision arthroplasty operation. The instrument removed from hip and after all of drainage and instrument cultures, empirically piperacillin/tazobactam started at the dose at $4 \mathrm{~g} / 500 \mathrm{mg}$ every eight hours. Pseudomonas Aeroginosa and Acinetobacter which both were sensitive to piperacillin/ tazobactam were isolated from drainage culture. Despite of potassium infusion, severe hypokalemia was developed in the patient. Potassium replacement was continued by parenteral and oral routes as $40-80 \mathrm{mEq} / \mathrm{day}$ but serum potassium level remained between $2.6-3.3 \mathrm{mEq} / \mathrm{L}$. Laboratory parameters were as follows: Glucose: $112 \mathrm{mg} / \mathrm{dL}$, Creatinin: $0.8 \mathrm{mg} / \mathrm{dL}$, BUN: $11.8 \mathrm{mg} / \mathrm{dL}$, Uric acid: $3.5 \mathrm{mg} / \mathrm{dL}, \mathrm{Na}: 140$ mEq/L, K: 3.3 mEq/L, Cl: 105 mEg/L, Ca: $7.1 \mathrm{mg} / \mathrm{dL}, \mathrm{P}: 3.8$ mg/dL, Mg: 1.9 mg/dL, AST: 32 U/L, ALT: 7 U/L, ALP: 144 U/L, GGT: 37 U/L, LDH: $431 \mathrm{U} / \mathrm{L}$; Total Protein: $6.5 \mathrm{gr} / \mathrm{dL}$, Albumin: $2.6 \mathrm{gr} / \mathrm{dL}$. Risk factors underlying hypokalemia were investigated. There were no medication (i.e. laxatives, diuretics, and aminoglycosides) and other disorder underlying the finding of resistant hypokalemia could be found. It was attributed to piperacillin treatment. Piperacillin/tazobactam therapy was replaced with ciprofloxacin therapy. Serum potassium level returned to normal ranges without potassium replacement (Fig. 1).

\section{Discussion}

Hypokalemia is serum potassium level less than $3.5 \mathrm{mEq} / \mathrm{L}$ 


\section{$K \mathrm{mmol} / \mathrm{L}$}

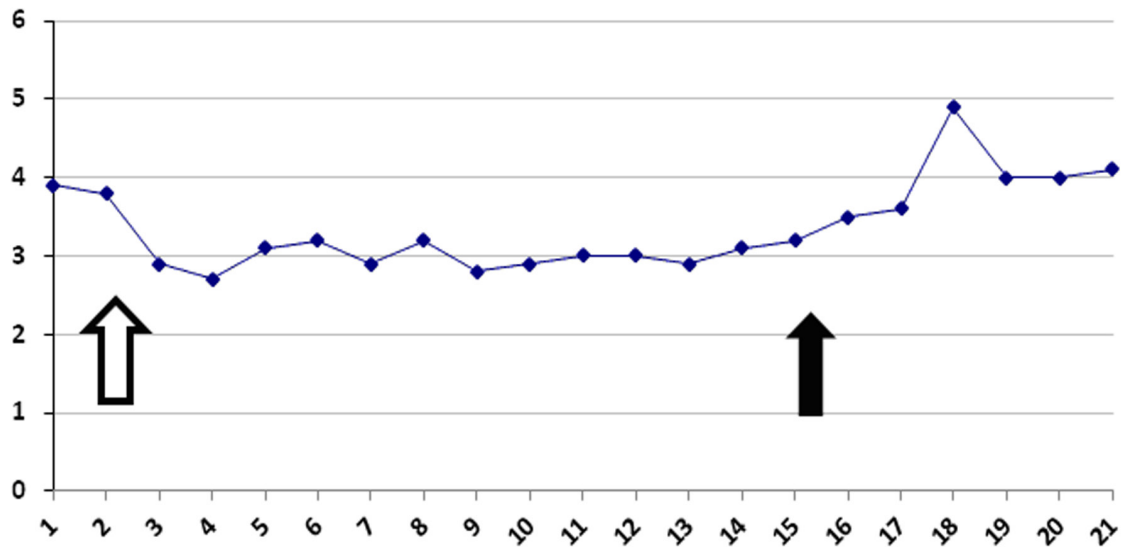

Days

Figure 1. Serum potassium level during the antibiotic therapy: White line shows the beginning and black line shows the end of piperacilline/tazobactam administration. Potassium replacement had continued during antibiotic therapy.

and is a common electrolyte abnormality in clinical practice. Potassium homeostasis is determined by kidney, and excess potassium is excreted in the urine. The normal range for serum potassium level is $3.5-5 \mathrm{mEq} / \mathrm{L}$.

Hypokalemia may result from conditions as varied as trans-cellular shift (movement of potassium from serum into cells as a result of insulin use or alkalosis), malnutrition or decreased dietary intake and parenteral nutrition, renal losses such as renal tubular acidosis, Bartter syndrome, Fanconi syndrome, hyperaldosteronism, magnesium depletion, leukemia, Cushing syndrome, gastrointestinal losses such as vomiting, pyloric stenosis, diarrhea, enemas or laxative use, gastric aspiration, ileal loop and medication effects $[1,2]$. Many drugs may lead to hypokalemia for example diuretics (most common cause), beta-adrenergic agonists, theophylline, steroids, and aminoglycosides [2,3]. The drugs can lead to hypokalemia in the therapeutic and toxic doses [3]. In our case there were no trans-cellular shift, no renal or gastrointestinal losses. Dietary intake was normal. We noticed that hypokalemia had begun after piperacillin/tazobactam administration. When the piperacillin/tazobactam was stopped, hypokalemia had resolved even without potassium replacement.

Piperacillin sodium exerts bactericidal activity by inhibiting septum formation and cell wall synthesis. In vitro, piperacillin is active against a variety of gram-positive and gram-negative aerobic and anaerobic bacteria. Tazobactam sodium is a $\beta$-lactamase inhibitor. Tazobactam, in combination with piperacillin enhances and extends the antibiotic spectrum of piperacillin to include $\beta$-lactamase producing bacteria normally resistant to piperacillin [1]. Its' known ad- verse effects are, mild to moderate in severity and transient nature, hypersensitivity reactions such as rush and pruritus; gastrointestinal system disorders such as diarrhea, nausea and vomiting; haematological disorders such as thrombocytopenia and neutropenia and rarely haemolytic anemia, hepatotoxicity, electrolyte and acid-base disturbances.

Piperacillin is excreted by both renal and biliary mechanisms, but the primary route of elimination is by glomerular filtration. Polderman et al [1], reported that treatment with piperacillin may cause or aggravate electrolyte disorders and tubular dysfunction in intensive care unit patients even when serum creatinine levels remain normal. Zaki et al [4], reported that a case of a 2-year-old girl who developed hypokalemic metabolic alkalosis and bradycardia after receiving intravenous piperacillin/tazobactam for bronchopneumonia. Hypokalemia and nephrotoxicity rate was increased in the patients with neutropenic fever when combination antibiotic therapy (such as aminoglycosid and piperacillin) was used.

In individuals with liver diseases or those receiving cytotoxic therapy or diuretics, piperacillin/tazobactam has been reported rarely to produce a decrease in serum potassium levels at high doses of piperacillin. There were no different causes that facilitated hypokalemia such as hepatic or renal failure in our patient. The usual total daily dose of piperacillin sodium/tazobactam sodium for adults is $12 \mathrm{~g} / 1.5 \mathrm{~g}$, given as $3 \mathrm{~g} / 0.375 \mathrm{~g}$ every six hours. In our case, hypokalemia was seen after 1 day of intravenous piperacillin/tazobactam administration at doses of $4 \mathrm{~g} / 500 \mathrm{mg}$ every eight hours. Our patient had no renal failure and dosage was appropriate for our patient. Piperacillin/tazobactam was monotherapy in our patient, none of aminoglycoside or other antibiotics were 
administered. Similarly, Hussein et al [5], reported that severe hypokalemia secondary to piperacillin/tazobactam in a patient with normal renal function. We attributed the piperacillin induced hypokalemia due to tubular dysfunction. Because when piperacillin stopped hypokalemia resolved immediately. Moreover there was no underlying renal dysfunction, other medications and comorbidities.

We also evaluated the causality of the case report. We concluded this causality as probable/likely category according to WHO-UMC Causality Categories because of the reasonable time between hypokalemia development and drug exposure and there was no relationship to other disease or drugs. Hypokalemia was resolved after discontinuation of drug.

In conclusion we must keep in the mind that even if renal and hepatic functions normal and in the absence of other medications such as diuretic laxatives etc., piperacillin/tazobactam may be cause severe hypokalemia and related life threating complications such as cardiac arrhythmia. Periodic electrolyte assessment should be performed in patients who are receiving piperacillin/tazobactam.

\section{Conflict of Interest}

Authors declare no conflict of interest.

\section{References}

1. Polderman KH, Girbes AR. Piperacillin-induced magnesium and potassium loss in intensive care unit patients. Intensive Care Med. 2002;28(4):520-522.

2. Kokot F, Hyla-Klekot L. Drug-induced abnormalities of potassium metabolism. Pol Arch Med Wewn. 2008;118(7-8):431-434.

3. Ben Salem C, Hmouda H, Bouraoui K. Drug-induced hypokalaemia. Curr Drug Saf. 2009;4(1):55-61.

4. Zaki SA, Lad V. Piperacillin-tazobactam-induced hypokalemia and metabolic alkalosis. Indian J Pharmacol. 2011;43(5):609-610.

5. Hussain S, Syed S, Baloch K. Electrolytes imbalance: a rare side effect of piperacillin/ tazobactam therapy. J Coll Physicians Surg Pak. 2010;20(6):419-420. 